\title{
Heat stress modulates the GSK-3 $\beta$ levels and Tau phosphorylation
}

\section{Tushar Dubey ${ }^{1,2}$ and Subashchandrabose Chinnathambi ${ }^{1,2, *}$}

\author{
${ }^{1}$ Neurobiology Group, Division of Biochemical Sciences, CSIR-National Chemical \\ Laboratory, Dr. Homi Bhabha Road 411008 Pune, India \\ ${ }^{2}$ Academy of Scientific and Innovative Research (AcSIR), 411008 Pune, India
}

\begin{abstract}
*To whom correspondence should be addressed: Prof. Subashchandrabose Chinnathambi, Neurobiology group, Division of Biochemical Sciences, CSIR-National Chemical Laboratory (CSIR-NCL), Dr. Homi Bhabha Road, 411008 Pune, India, Telephone: +91-20-25902232, Fax.+91-20-25902648. Email: s.chinnathambi@ncl.res.in.
\end{abstract}

\begin{abstract}
Alzheimer's disease is a prominent neurological disorder, which leads to progressive dementia. The microtubule-associated protein Tau is been considered as one of the major causes of Alzheimer's disease. Physiologically Tau assists in the stabilization of microtubules, contrary to this the pathological state of Tau results in the formation of neurotoxic tangles of Tau. The posttranslational modifications, such as GSK-3 $\beta$-mediated Tau phosphorylation results in the generation of Tau pathology. Neuroinflammation generated in Alzheimer's disease, contributes to elevated body temperature. The aim of present work is to study the effect of high temperature on Tau phosphorylation. The neuroblastoma cells were exposed to heat stress for 40 minutes. The immunofluorescence and western blot studies suggested that high temperature increases the levels of GSK-3 $\beta$ in cells. Heat stressed cells was also observed to have elevated levels of phosphorylated Tau. Additionally, heat stressed cells found to have modulated nuclear transport as the level of Ran was reduced. The results of present work suggested that increased temperature could be considered as a risk factor in Alzheimer's disease as it elevated the GSK-3 $\beta$ levels in cells thus, resulting in increased Tau phosphorylation.
\end{abstract}

\section{Keywords}

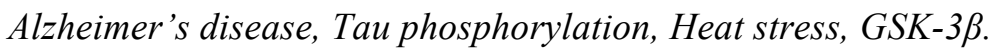




\section{Introduction}

Alzheimer's disease is the neurodegenerative disorder, resulting in symptoms-like memory loss, behavioural impairment and locomotory deficits ${ }^{1,2}$. Alzheimer's disease is hallmarked by two types of protein aggregates, which are senile plaques composed of amyloid- $\beta$ peptide and neurofibrillary tangles (NFTs) composed up of microtubule-associated protein Tau ${ }^{3,4}$. The action of gamma-secretase on amyloid precursor protein (APP) results in the generation of amyloid $\beta-42$ peptides, leading to generation of extracellular senile plaques ${ }^{5}$. Tau protein is considered to be a cytoskeletal protein, having role in stabilization of the microtubules ${ }^{6-8}$. In the pathological state Tau detaches from microtubules and lead to the formation of intracellular neurofibrillary tangle NFTs ${ }^{9}{ }^{10}$. Tau is a 441 amino acid long natively unfolded protein, the domain organization of Tau comprises of projection domain and microtubule-binding domain ${ }^{10,11}$. The four repeat region present in microtubule-binding domain is associated with the Tau pathology ${ }^{12}$. The four repeat region is prone to several posttranslational modifications including phosphorylation, glycation, glycosylation etc. Tau phosphorylation is one of the major cause of pathologic state of $\mathrm{Tau}^{13}$. Tau phosphorylation contributes to several defects in cell signalling leading to neurotoxicity ${ }^{14}$. The increased load of Tau phosphorylation leads to generation intracellular $\operatorname{ROS}^{15}$. Heavy metal such as mercury and iron are known to modulate Tau phosphorylation ${ }^{16}$. Similarly, abnormal phosphorylation of Tau results in reduced microtubule binding of Tau ${ }^{17}$. Thus, phospho-Tau and the Tau specific kinases are considered as a prime target for therapeutic studies in AD. GSK-3 $\beta$ and CDK5 are the principle Ser/Thr kinases, while Src family kinases (SFKs), FYN and the ABL family kinases phosphorylate tyrosine ${ }^{18}$. Several compound such as leptin, which downregulates Tau phosphorylation are designated as potent molecules against $\mathrm{AD}^{19}$. Similarly metal ions as Lithium also showed promising results in reducing Tau phosphorylation by inhibiting GSK- $3 \beta^{20}$. GSK-3 $\beta$ and CDK 5 phosphorylates Tau on various sites which ultimately results in Tau pathology ${ }^{21}$. Additionally, these kinases are also reported to phosphorylate the GPCR proteins, which contributes to AD pathology ${ }^{22}$. GSK-3 $\beta$ gets phosphorylated at Ser-9 leading to generation of its inactive state pGSK3- $\beta^{23}$. Several studies have suggested increased levels of GSK-3 $\beta$ in AD condition. Fluctuation of body temperature has been observed in AD patients. Hypothermia has also been marked as a risk factor for AD. The published studies have suggested that hypothermia might down regulate the GSK- $3 \beta$ expression ${ }^{24}$. On contrary the reports suggested that as a result of neuroinflammation AD patient may suffer increased body temperature ${ }^{25}$. The deregulation of nucleocytoplasmic transport is one of the consequences of neurodegeneration. Reduced levels of Ran has been observed in case of neurodegeneration ${ }^{26}$. Similarly the deformities in the arrangement of nuclear pore complex (Nups) also studied in neurodegenerative disease ${ }^{27}$. The aim of our work is to study the effect of increased temperature on GSK-3 $\beta$ levels and Tau phosphorylation. The work was intended to investigate the fact weather increased body temperature could be one of risk factors for $\mathrm{AD}$ patients. 


\section{Results}

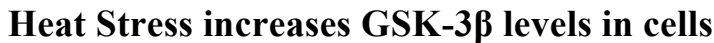

GSK-3 $\beta$ is the Ser/Thr kinase, which targets microtubule-associated protein Tau. The increased phosphorylation of Tau leads to aggregation. Thus, GSK-3ßis considered to be associated with Alzheimer's disease. Neuroinflammation results in elevated body temperature in Alzheimer's disease $(\mathrm{AD})^{28}$. Here we observed the effect of high temperature on GSK-3 $\beta$ levels in neuronal cells. Earlier studies have suggested that at temperature of $43^{\circ} \mathrm{C}$ the cells experiences the heat stress leading to generation of several stress responses ${ }^{29}$. Thus in our studies, the cells were incubated at $43^{\circ} \mathrm{C}$ for 40 minutes to induce the heat stress (Figure 1A). The immunofluorescence studies suggested that heat

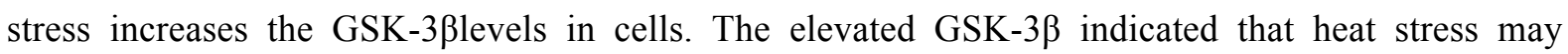
contribute to increased levels of Tau phosphorylation (Figure 1B). The quantification of immunofluorescence images supported the results that intensity of GSK-3 $\beta$ increased after exposure

\section{Figure 1}

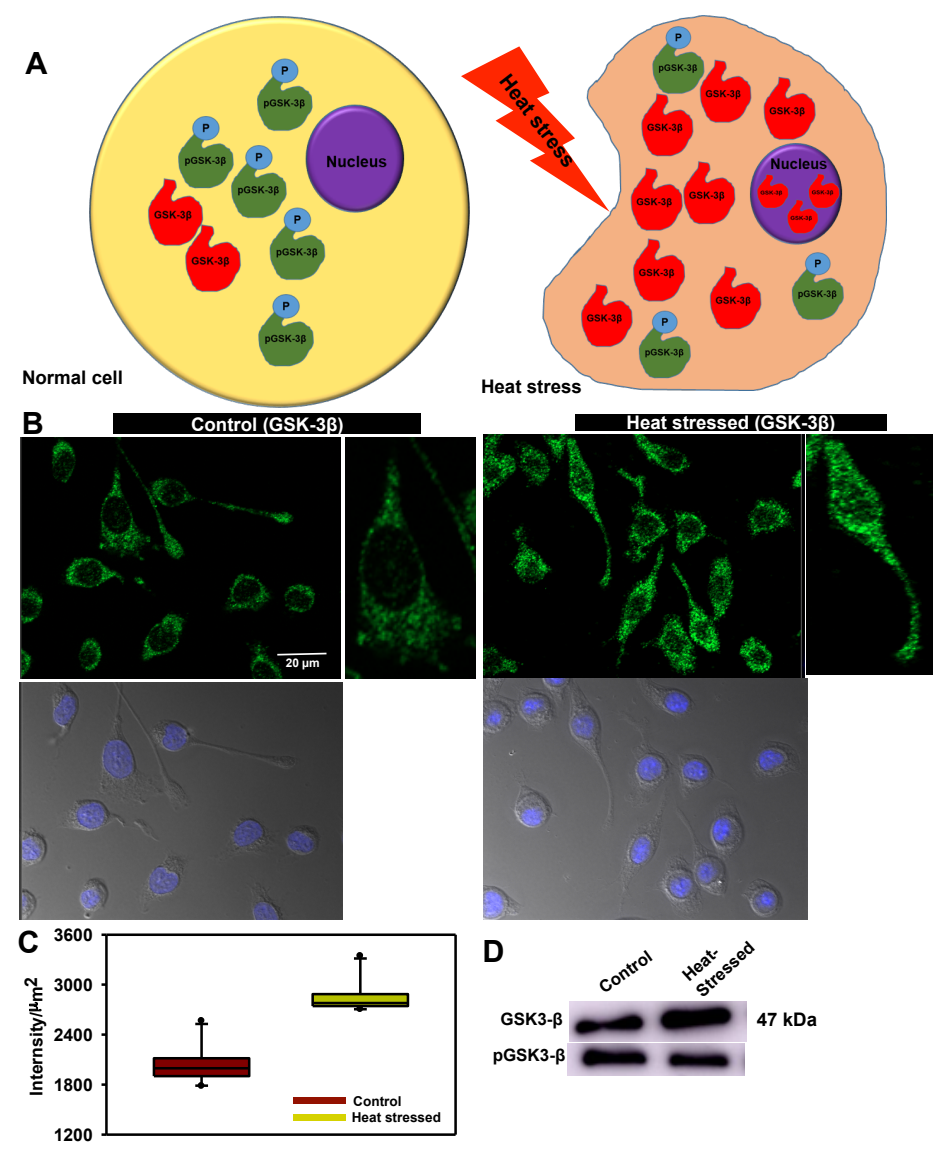

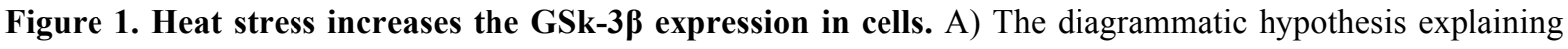
the effect of heat stress in cells. B) The immunoblot suggested that heat stress at $43^{\circ} \mathrm{C}$ for 40 minutes increases the expression of GSK-3 $\beta$ in cells. C) The quantification of immunofluorescence images showing the increased 


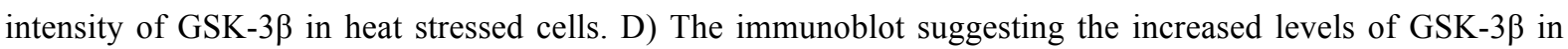
heat stress cells, whereas pGSK-3 $\beta$ levels remains unaltered as compared to control.

to high temperature. The western blot analysis of heat stressed cells suggested that the levels of GSK$3 \beta$ elevated after heat stress, whereas no changes in pGSK-3 $\beta$ levels were observed. Thus, the above results indicated that heat stress might elevate the levels of GSK3- $\beta$, which could be reason for induction of Tau pathology.

\section{Figure 2}

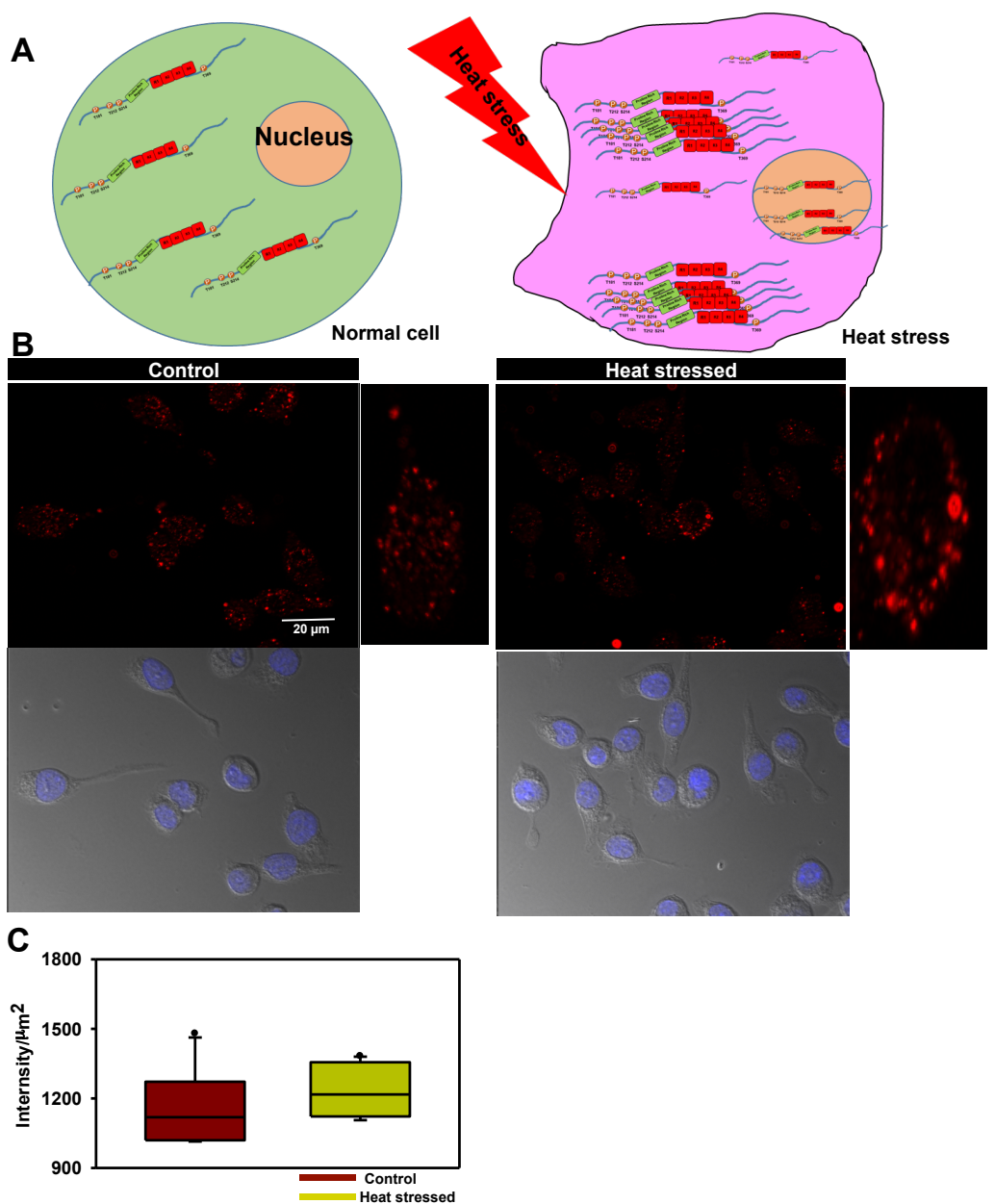

Figure 2. Heat stress increases the phospho-Tau in cells. A) The diagrammatic explanation of effect of heat stress on level of phospho-Tau in cell. B) The immunofluorescence images sowing the presence of phosphoTau (AT100) in heat stressed cell as compared to control cells. C) The quantification of immunofluorescence images indicating increased intensity of AT100 in heat stressed cells as compared to control cells.

\section{Heat Stress elevates phospho-Tau levels in cells}

Several factors contribute to the generation of Tau pathology, among these phosphorylation of Tau is considered as leading cause of Tau pathology. Tau protein has 85 sites which are targeted by various kinases for of phosphorylation. GSK-3 $\beta$, which phosphorylates Tau, at sites such as S199, S202, 
T231, S235, S396, S413 etc., resulting in its pathological state. In this study we observed the effect of increased temperature on levels of phospho-Tau in cells (Figure 2A). The immunofluorescence images suggested that the cells incubated at $43^{\circ} \mathrm{C}$ for 40 minutes showed increased levels of phosphoTau (Ser212/214) (Figure 2B). The quantification of immunofluorescence images supported the fact that intensity of phospho-Tau was elevated in heat stressed cells (Figure 2C). The elevated levels of phospho-Tau in heat stressed cells indicated that increase in temperature could be one of the risk factors for Tauopathy.

\section{Figure 3}

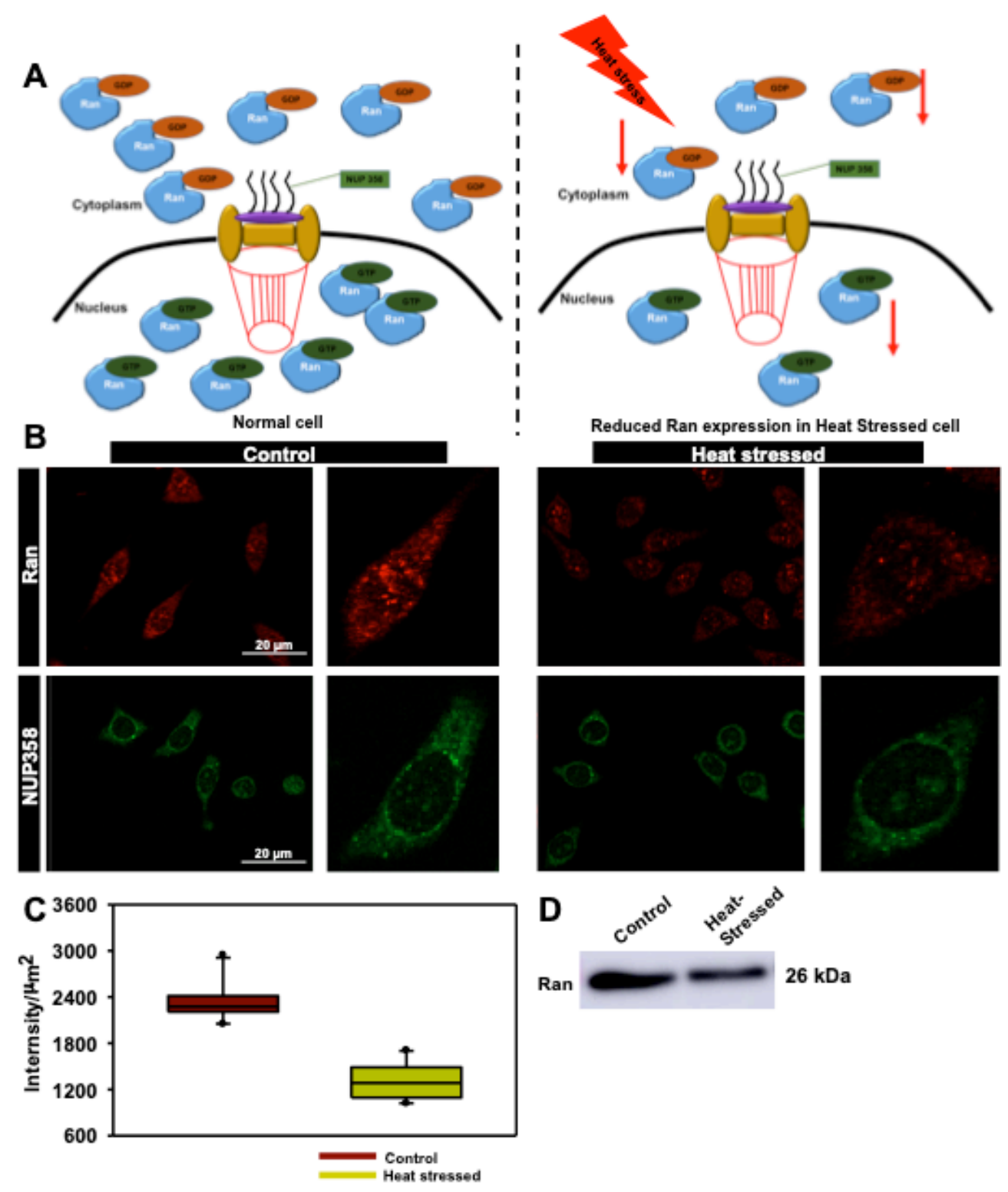

Figure 3. Heat stress modulates nuclear transport. A) The diagrammatic representation of nuclear transport of normal cells and heat stressed cells. B) The immunofluorescence images showing the effect of heat stress on nuclear transport. Ran gradient was reduced in the heat stress cells, whereas the NUP358 morphology was 
unaltered. C) The quantification of immunofluorescence images suggests the reduced level of Ran in heat stress cells. D) The immuno blot analysis of heat stressed cells vs. control cells. The heat stress cells was observed to have low level of Ran as compared to control.

\section{The effect of high temperature on nucleocytoplasmic transport}

The nucleocytoplasmic transport in cells is govern by several carrier proteins. Ras-associated nuclear protein (Ran) is the key protein for nucleocytoplasmic transport. Recent studies suggested that under neurodegenerative condition the Ran gradient gets disturbed, which results in neuronal death ${ }^{30}$. The objective of our study was to observe the effect of high temperature on nucleocytoplasmic transport. (Figure 3A). The immunofluorescence images indicated that the levels of Ran were reduced in heat stressed cells. The modulation in Ran levels could be due to disturbed nuclear transport (Figure 3B). The quantification results suggested that the intensity of Ran lowers down in the heat stress cells (Figure 3C). The immunoblot analysis suggested that the level of Ran protein were decreased in heat stressed cells (Figure 3D). On contrary, we observed no modulation in NUP358 arrangement in heat stressed cells. Thus, these studies evidenced that elevated temperature could modulate the nucleocytoplasmic transport, resulting in the generation of neurodegeneration.

\section{Discussion}

The neurodegeneration includes diseases such as include Alzheimer's disease, fronto-temporal dementia, Parkinson's disease, vascular dementia, lewy body dementia, Posterior Cortical Atrophy, Creutzfeldt-Jakob Disease etc ${ }^{31-33}$. The protein aggregates of amyloid- $\beta-42$ and the microtubuleassociated protein Tau are considered as the major cause of Alzheimer's disease ${ }^{6,13}$. AD is symptomized by a spectrum of symptoms including impaired cognition, memory deprivation, emotional imbalance and problem in performing routine activities ${ }^{9}$. Under the pathological condition, the hyperphosphorylation of Tau leads to the generation of several structural and functional modifications resulting in neurotoxicity ${ }^{34}$. Cells have various classes of kinases, which include serine/threonine kinase and tyrosine kinase ${ }^{34,35}$. GSK-3 $\beta$ and CDK5 prominent Tau targeting kinases $\mathrm{Tau}^{36}$, which are considered to be involved in generation of Tau pathology ${ }^{37}$. Several studies claim the involvement of GSK-3 $\beta$ in AD pathology, which includes memory impairment, locomotry dysfunction and behavioural impairments ${ }^{38}$. The amyloid- $\beta$-induced neurotic damage have been considered to be mediated by GSK-3 $\beta$ activation ${ }^{39}$. Similarly, increased levels of GSK-3 $\beta$ was observe in the brain of AD patients ${ }^{40}$. Recent studies suggested that the silencing of GSK-3 $\beta$ reduces the tangles formation in mouse model of $\mathrm{AD}^{41}$. The selective GSK-3 $\beta$ inhibitor SAR502250 was reported to reduce the behavioural impairment and neuropsychiatric symptoms in rodent $A D$ models $^{42}$. GSK-3 $\beta$ is leads to apoptotic cell death by inhibiting PI3K pathways ${ }^{43}$. In addition to AD, GSK-3 $\beta$ is studied to be involved in other Tau related-frontotemporal dementia ${ }^{38}$. GSK3- $\beta$, also 
induces the caspase activity in endoplasmic reticulum stressed cells ${ }^{44}$. Additionally, GSK-3 $\beta$ modulate the Nrf2 signalling pathway under the stress conditions ${ }^{45}$. During bacterial infection GSK-3 $\beta$ modulated the NF- $\mathrm{KB}$ levels leading to inflammation ${ }^{46}$. As GSK-3 $\beta$ is reported to be involved in AD pathology, several strategies have been applied to inhibit the GSK-3 $\beta$. Indirubins and derivatives showed potent inhibition of GSK-3 $\beta^{47}$. Similarly erroneous, alsterpaullone, purvalanol,

\section{Figure 4}

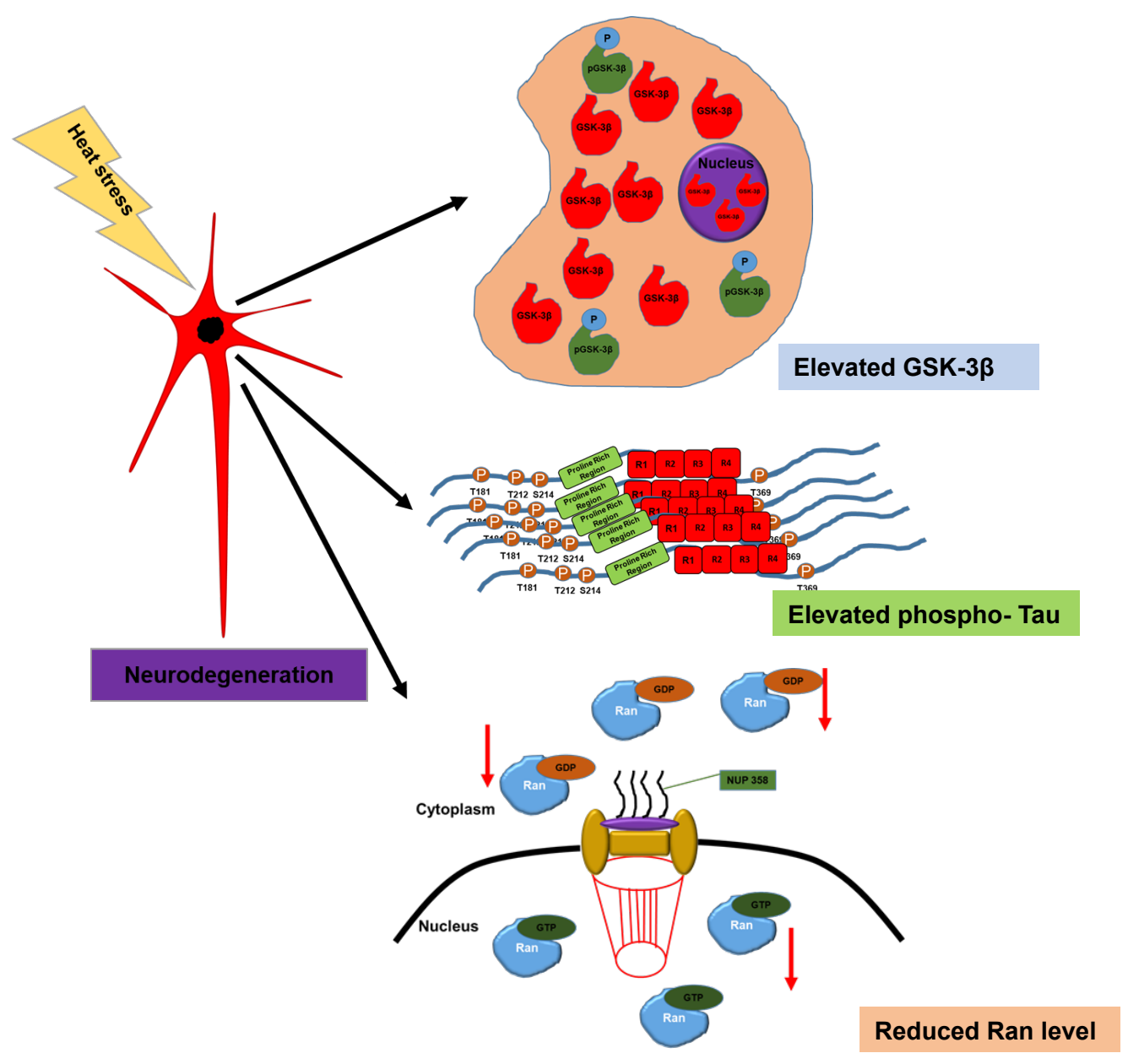

Figure 4. The diagrammatic representation of our hypothesis which suggests that heat stress modulate the GSK$3 \beta$ levels in cells. The increased GSK-3 $\beta$ in cells contributes to the elevated load of phospho-Tau, which leads to various cell toxicity. Heat stress was found to reduce the Ran level in cells whereas Nup358 was observed to be altered.

pyrazolopyrimidine are also the compounds studied to have effect in downregulation of GSK- $3 \beta^{48}$. The hypothermia in patient with neurological disorders have been reported to modulate the GSK-3 $\beta$ levels ${ }^{24,49}$. The AD patients also suffer the increased core body temperature as a result of activated inflammatory pathways ${ }^{25}$. The effect of high temperature on GSK-3 $\beta$ levels were not studied yet. Here we observed that heat stress increased the GSK-3 $\beta$ expression in cells, which ultimately led to 
increased phospho-Tau levels in neurons. Ran is one of the key protein of nuclear transport ${ }^{50}$. Phospho-Tau has also been reported to disrupt the nuclear transport by reducing the Ran gradient in cells ${ }^{30}$. In our study we have also monitored that heat stress results in reduction of Ran levels in cells indicating the impaired nucleocytoplasmic transport. Thus, we suggest that heat stress could act as risk factor in $\mathrm{AD}$ which ultimately led to Tau hyperphosphorylation.

\section{Conclusion}

Tau phosphorylation is considered as a major cause of Tau pathology. The GSK-3 $\beta$ is associated with Tau phosphorylation. The results of present work suggest us that high temperature could increase the expression of GSK-3 $\beta$ and phospho-Tau in neurons. Additionally, heat stress modulated the nuclear transport by the reducing level of Ran although the Nup358 arrangement and nuclear morphology was observed to be unaltered. Thus, the overall results suggest that high temperature could contribute to $\mathrm{AD}$ pathology and hence, it could be considered as a risk factor for AD.

\section{Materials and Methods}

\section{Chemicals and Reagents}

Triton X-100 was purchased from Sigma. DMEM advanced F12 media (12634010), fetal bovine serum (1600004), penstrep cocktail (10378016), anti-fungal agent Anti-anti (15240062) were purchased from Gibco. GSK-3 $\beta$ monoclonal antibody, AT100 (MN1060), Goat anti-mouse Alexa fluor plus 555 (A32727), Goat anti-rabbit Alexa IgG fluor 488 (A-11008) antibodies were purchased from Thermo fisher. Ran (ab11693) antibody was purchased from Abcam. The secondary antibodies Goat anti-Rabbit HRP conjugated and Goat anti-mouse HRP conjugated were purchased from Thermo fisher, Clarity ${ }^{\mathrm{TM}}$ Western ECL Substrate (1705060 - Bio-Rad).

\section{Immunoblot assay}

The mouse neuroblastoma cells Neuro2a (ATCC CCL-131) were acquired from ATCC. The cells were maintained in advanced DMEM/F-12 media supplemented with $10 \%$ fetal bovine serum. The cells were kept in incubator at $37^{\circ} \mathrm{C}$ supplied with $5 \% \mathrm{CO}_{2}$. The cells were regularly passaged and maintained.

The effect of heat stress were studied in mouse neuroblastoma cells (Neuro2a cells). For the western blot analysis neuronal cells were seeded at density of $1.5 \times 10^{5}$ cells/well in 12 well poly-lysine coated plate. The cells were subjected to heat stress at $43^{\circ} \mathrm{C}$ for 40 minutes. The cells were lysed with RIPA buffer supplemented with protease inhibitor cocktail. The lysate was centrifuged at $12000 \mathrm{rpm}$ for 20 
minutes at $4{ }^{\circ} \mathrm{C}$ and the supernatant was collected and loaded on $10 \%$ SDS-PAGE. The protein was transferred to PVDF membrane using semi-dry blot transfer unit (GE). The blots were incubated with $5 \%$ skimmed milk for 60 minutes at room temperature for blocking the nonspecific sites. Following blocking, the blots were incubated with specific primary antibodies viz. GSK-3 $\beta(1: 1000)$, pGSK$3 \beta(1: 1000)$, AT100 (1:1000), Ran $(1: 10,000)$. The blots were incubated with primary antibody at $4{ }^{\circ} \mathrm{C}$ for overnight. The blots were washed thrice with PBST, pH 7.4 and incubated with HRP-conjugated secondary antibody for 1 hour at room temperature. The blots were washed trice with PBST and developed using clarity max ${ }^{\circledR}$ Bio-Rad ECL solution. The blots were imaged in Amersham AI600 chemiluminescent imager. Each set of experiment were repeated twice and the quantification was done using ImageJ software.

\section{Immunofluorescence}

The immunofluorescence studies were further carried out to validate the effect of heat stress on various cellular signalling. Neuro2a cells $(25,000$ cells/well) were divided into two groups, group one comprised of cells exposed to heat stress and second group cells were kept at $37^{\circ} \mathrm{C}$, which was designated as cell control. After the incubation, the cells were washed with 1X PBS, followed by $4 \%$ paraformaldehyde to fix the cells. The cells were permeabilized using $0.2 \%$ Triton X-100 followed by blocking with $2 \%$ horse serum for $60 \mathrm{~min}$. The cells were incubated overnight with monoclonal primary antibodies GSK-3 $\beta$ (1:100), AT100 (1:100), Ran (1:1000), NUP $358(1: 1000)$ at $4^{\circ} \mathrm{C}$. After three subsequent washes of $1 \mathrm{X}$ PBS, the cells were incubated with secondary antibodies goat antirabbit Alexa 488 (A11034) and goat anti-mouse Alexa 555 (A32727) for 60 minutes at $37^{\circ} \mathrm{C}$. After incubation the cells were washed with 1 X PBS and $300 \mathrm{nM}$ of DAPI was added onto cells. After the final wash with $1 \mathrm{X}$ PBS, the coverslips were mounted on slides (blue star) and sealed. The samples were imaged on Zeiss Axio Observer 7 with Apotome 2.0. The mages were quantified in Zen 2 (Blue) software and the statistics were plotted using SigmaPlot 10.0.

\section{Statistical analysis}

The statistical data for the fluorescence measurement or viability assay was plotted by using either duplicate or triplicate reading. Untransformed (raw) data were analysed and plotted by SigmaPlot 10.0 software. The data was analysed for the significance by Student's $t$-test.

\section{Competing Interest}

The authors declare that they have no competing interest.

\section{Funding}

This project is supported by grant from the in-house, National Chemical Laboratory-Council of Scientific Industrial Research-(CSIR-NCL) MLP029526.

\section{Author Contributions}


S.C, T.D designed and carried out the experiments. T.D and S.C analyzed the data. S.C. conceived the idea of the project, provided resources, supervised and wrote the manuscript. All authors contributed to the discussions and manuscript review.

\section{Acknowledgements}

The authors acknowledge CSIR-National Chemical Laboratory for the instrumentation central facility. Tushar Dubey acknowledges the fellowship from University of Grant Commission (UGC), India.

\section{Author Information}

\section{Tushar Dubey and Subashchandrabose Chinnathambi}

Neurobiology Group, Division of Biochemical Sciences, CSIR-National Chemical Laboratory, Dr. Homi Bhabha Road, 411008 Pune, India

\section{Tushar Dubey and Subashchandrabose Chinnathambi}

Academy of Scientific and Innovative Research (AcSIR), 110025 New Delhi, India

\section{Corresponding Author}

Correspondence and requests for materials should be addressed to Prof. Subashchandrabose

Chinnathambi. Email: s.chinnathambi@,ncl.res.in

Telephone: +91-20-25902232, Fax. +91-20-25902648.

\section{ORCID}

Subashchandrabose Chinnathambi: 0000-0002-5468-2129

\section{References}

1. Balmik, A. A.; Chinnathambi, S., Multi-faceted role of melatonin in neuroprotection and amelioration of Tau aggregates in Alzheimer's disease. Journal of Alzheimer's Disease 2018, 62 (4), 1481-1493.

2. Das, R.; Balmik, A. A.; Chinnathambi, S., Phagocytosis of full-length Tau oligomers by Actin-remodeling of activated microglia. Journal of Neuroinflammation 2020, 17 (1), 1-15.

3. Ittner, L. M.; Götz, J., Amyloid- $\beta$ and tau-a toxic pas de deux in Alzheimer's disease. Nature Reviews Neuroscience 2011, 12 (2), 67.

4. Sonawane, S. K.; Balmik, A. A.; Boral, D.; Ramasamy, S.; Chinnathambi, S., Baicalein suppresses Repeat Tau fibrillization by sequestering oligomers. Archives of Biochemistry and Biophysics 2019, 675, 108119.

5. Sakono, M.; Zako, T., Amyloid oligomers: formation and toxicity of $\mathrm{A} \beta$ oligomers. The FEBS journal 2010, 277 (6), 1348-1358.

6. Sonawane, S. K.; Ahmad, A.; Chinnathambi, S., Protein-Capped Metal Nanoparticles Inhibit Tau Aggregation in Alzheimer's Disease. ACS Omega 2019, 4 (7), 12833-12840.

7. Gorantla, N. V.; Das, R.; Balaraman, E.; Chinnathambi, S., Transition metal nickel prevents Tau aggregation in Alzheimer's disease. International journal of biological macromolecules 2019.

8. Gorantla, N. V.; Landge, V. G.; Nagaraju, P. G.; Priyadarshini CG, P.; Balaraman, E.; Chinnathambi, S., Molecular cobalt (II) complexes for tau polymerization in Alzheimer's disease. ACS omega 2019, 4 (16), 16702-16714.

9. Mandelkow, E.-M.; Mandelkow, E., Biochemistry and cell biology of tau protein in neurofibrillary degeneration. Cold Spring Harbor perspectives in medicine 2012, a006247. 
10. Desale, S. E.; Chinnathambi, S., Role of dietary fatty acids in microglial polarization in Alzheimer's disease. Journal of Neuroinflammation 2020, 17 (1), 1-14.

11. Mandelkow, E.-M.; Mandelkow, E., Tau in Alzheimer's disease. Trends in cell biology 1998, $8(11), 425-427$.

12. Balmik, A. A.; Das, R.; Dangi, A.; Gorantla, N. V.; Marelli, U. K.; Chinnathambi, S., Melatonin interacts with repeat domain of Tau to mediate disaggregation of paired helical filaments. Biochimica et Biophysica Acta (BBA)-General Subjects 2019, 129467.

13. Sonawane, S. K.; Chinnathambi, S., Prion-Like Propagation of Post-Translationally Modified Tau in Alzheimer's Disease: A Hypothesis. Journal of Molecular Neuroscience 2018, 65 (4), 480490.

14. Hanger, D. P.; Anderton, B. H.; Noble, W., Tau phosphorylation: the therapeutic challenge for neurodegenerative disease. Trends in molecular medicine 2009, 15 (3), 112-119.

15. Dias-Santagata, D.; Fulga, T. A.; Duttaroy, A.; Feany, M. B., Oxidative stress mediates tauinduced neurodegeneration in Drosophila. The Journal of clinical investigation 2007, 117 (1), 236245 .

16. Egaña, J. T.; Zambrano, C.; Nuñez, M. T.; Gonzalez-Billault, C.; Maccioni, R. B., Ironinduced oxidative stress modify tau phosphorylation patterns in hippocampal cell cultures. Biometals 2003, $16(1), 215-223$.

17. Bramblett, G. T.; Goedert, M.; Jakes, R.; Merrick, S. E.; Trojanowski, J. Q.; Lee, V. M., Abnormal tau phosphorylation at Ser396 in Alzheimer's disease recapitulates development and contributes to reduced microtubule binding. Neuron 1993, 10 (6), 1089-1099.

18. Nygaard, H. B., Targeting Fyn kinase in Alzheimer's disease. Biological psychiatry 2018, 83 (4), 369-376.

19. Greco, S. J.; Sarkar, S.; Johnston, J. M.; Zhu, X.; Su, B.; Casadesus, G.; Ashford, J. W.; Smith, M. A.; Tezapsidis, N., Leptin reduces Alzheimer's disease-related tau phosphorylation in neuronal cells. Biochemical and biophysical research communications 2008, 376 (3), 536-541.

20. Hong, M.; Chen, D. C.; Klein, P. S.; Lee, V. M.-Y., Lithium reduces tau phosphorylation by inhibition of glycogen synthase kinase-3. Journal of Biological Chemistry 1997, 272 (40), 2532625332.

21. Šimić, G.; Babić Leko, M.; Wray, S.; Harrington, C.; Delalle, I.; Jovanov-Milošević, N.; Bažadona, D.; Buée, L.; De Silva, R.; Di Giovanni, G., Tau protein hyperphosphorylation and aggregation in Alzheimer's disease and other tauopathies, and possible neuroprotective strategies. Biomolecules 2016, 6 (1), 6.

22. Chidambaram, H.; Chinnathambi, S., G-Protein coupled receptors and tau-different roles in alzheimer's disease. Neuroscience $\mathbf{2 0 2 0}$.

23. Agarwal-Mawal, A.; Qureshi, H. Y.; Cafferty, P. W.; Yuan, Z.; Han, D.; Lin, R.; Paudel, H. K., 14-3-3 connects glycogen synthase kinase-3 $\beta$ to tau within a brain microtubule-associated tau phosphorylation complex. Journal of Biological Chemistry 2003, 278 (15), 12722-12728.

24. Kelly, S.; Cheng, D.; Steinberg, G. K.; Yenari, M. A., Mild hypothermia decreases GSK3 $\beta$ expression following global cerebral ischemia. Neurocritical care 2005, 2 (2), 212-217.

25. Klegeris, A.; Schulzer, M.; Harper, D. G.; McGeer, P. L., Increase in core body temperature of Alzheimer's disease patients as a possible indicator of chronic neuroinflammation: a meta-analysis. Gerontology 2007, 53 (1), 7-11.

26. Mastroeni, D.; Chouliaras, L.; Grover, A.; Liang, W. S.; Hauns, K.; Rogers, J.; Coleman, P. D., Reduced RAN expression and disrupted transport between cytoplasm and nucleus; a key event in Alzheimer's disease pathophysiology. PloS one 2013, 8 (1), e53349.

27. Sheffield, L. G.; Miskiewicz, H. B.; Tannenbaum, L. B.; Mirra, S. S., Nuclear pore complex proteins in Alzheimer disease. Journal of Neuropathology \& Experimental Neurology 2006, 65 (1), 45-54.

28. Eikelenboom, P.; Bate, C.; Van Gool, W.; Hoozemans, J.; Rozemuller, J.; Veerhuis, R.; Williams, A., Neuroinflammation in Alzheimer's disease and prion disease. Glia 2002, 40 (2), 232239.

29. Kirby, B. A.; Merril, C. R.; Ghanbari, H.; Wallace, W. C., Heat shock proteins protect against stress-related phosphorylation of tau in neuronal PC12 cells that have acquired thermotolerance. Journal of Neuroscience 1994, 14 (9), 5687-5693. 
30. Eftekharzadeh, B.; Daigle, J. G.; Kapinos, L. E.; Dujardin, S.; Coyne, A.; Miller, S. J.; Cook, C.; Grima, J. C.; Bennett, R. E.; Schiantarelli, J., Tau Protein Disrupts Nucleocytoplasmic Transport in Alzheimer's Disease. 2018.

31. Snowden, J. S.; Neary, D.; Mann, D. M., Frontotemporal dementia. The British journal of psychiatry 2002, 180 (2), 140-143.

32. Lang, A. E.; Lozano, A. M., Parkinson's disease. New England Journal of Medicine 1998, $339(16), 1130-1143$.

33. Gorantla, N. V.; Chinnathambi, S., Tau Protein Squired by Molecular Chaperones During Alzheimer's Disease. Journal of Molecular Neuroscience 2018, 66 (3), 356-368.

34. Augustinack, J. C.; Schneider, A.; Mandelkow, E.-M.; Hyman, B. T., Specific tau phosphorylation sites correlate with severity of neuronal cytopathology in Alzheimer's disease. Acta neuropathologica 2002, 103 (1), 26-35.

35. Ishiguro, K.; Sato, K.; Takamatsu, M.; Park, J.; Uchida, T.; Imahori, K., Analysis of phosphorylation of tau with antibodies specific for phosphorylation sites. Neuroscience letters $\mathbf{1 9 9 5}$, $202(1-2), 81-84$.

36. Buée, L.; Bussière, T.; Buée-Scherrer, V.; Delacourte, A.; Hof, P. R., Tau protein isoforms, phosphorylation and role in neurodegenerative disorders. Brain Research Reviews 2000, 33 (1), 95 130 .

37. Maqbool, M.; Mobashir, M.; Hoda, N., Pivotal role of glycogen synthase kinase-3: a therapeutic target for Alzheimer's disease. European journal of medicinal chemistry 2016, 107, 63-81. 38. Hernandez, F.; Lucas, J. J.; Avila, J., GSK3 and tau: two convergence points in Alzheimer's disease. Journal of Alzheimer's disease 2013, 33 (s1), S141-S144.

39. Hernández, F.; de Barreda, E. G.; Fuster-Matanzo, A.; Lucas, J. J.; Avila, J., GSK3: a possible link between beta amyloid peptide and tau protein. Experimental neurology 2010, 223 (2), 322-325.

40. Hooper, C.; Killick, R.; Lovestone, S., The GSK3 hypothesis of Alzheimer's disease. Journal of neurochemistry 2008, 104 (6), 1433-1439.

41. Hurtado, D. E.; Molina-Porcel, L.; Carroll, J. C.; MacDonald, C.; Aboagye, A. K.; Trojanowski, J. Q.; Lee, V. M.-Y., Selectively silencing GSK-3 isoforms reduces plaques and tangles in mouse models of Alzheimer's disease. Journal of Neuroscience 2012, 32 (21), 7392-7402.

42. Griebel, G.; Stemmelin, J.; Lopez-Grancha, M.; Boulay, D.; Boquet, G.; Slowinski, F.; Pichat, P.; Beeské, S.; Tanaka, S.; Mori, A., The selective GSK3 inhibitor, SAR502250, displays neuroprotective activity and attenuates behavioral impairments in models of neuropsychiatric symptoms of Alzheimer's disease in rodents. Scientific Reports 2019, 9 (1), 1-15.

43. Gómez-Sintes, R.; Hernández, F.; Lucas, J. J.; Avila, J., GSK-3 mouse models to study neuronal apoptosis and neurodegeneration. Frontiers in molecular neuroscience 2011, 4, 45.

44. Meares, G. P.; Mines, M. A.; Beurel, E.; Eom, T.-Y.; Song, L.; Zmijewska, A. A.; Jope, R. S., Glycogen synthase kinase-3 regulates endoplasmic reticulum (ER) stress-induced CHOP expression in neuronal cells. Experimental cell research 2011, 317 (11), 1621-1628.

45. Chowdhry, S.; Zhang, Y.; McMahon, M.; Sutherland, C.; Cuadrado, A.; Hayes, J. D., Nrf2 is controlled by two distinct $\beta$-TrCP recognition motifs in its Neh6 domain, one of which can be modulated by GSK-3 activity. Oncogene 2013, 32 (32), 3765-3781.

46. Jope, R. S.; Yuskaitis, C. J.; Beurel, E., Glycogen synthase kinase-3 (GSK3): inflammation, diseases, and therapeutics. Neurochemical research 2007, 32 (4-5), 577-595.

47. Polychronopoulos, P.; Magiatis, P.; Skaltsounis, A.-L.; Myrianthopoulos, V.; Mikros, E.; Tarricone, A.; Musacchio, A.; Roe, S. M.; Pearl, L.; Leost, M., Structural basis for the synthesis of indirubins as potent and selective inhibitors of glycogen synthase kinase-3 and cyclin-dependent kinases. Journal of medicinal chemistry 2004, 47 (4), 935-946.

48. Bain, J.; McLauchlan, H.; Elliott, M.; Cohen, P., The specificities of protein kinase inhibitors: an update. Biochemical Journal 2003, 371 (Pt 1), 199.

49. A Whittington, R.; Papon, M.-A.; Chouinard-Decorte, F.; Planel, E., Hypothermia and Alzheimer's disease neuropathogenic pathways. Current Alzheimer Research 2010, 7 (8), 717-725.

50. Moore, M. S., Ran and nuclear transport. Journal of Biological Chemistry 1998, 273 (36), 22857-22860. 